\title{
Discovery of the Next-Generation Pan-TRK Kinase Inhibitors for the Treatment of Cancer
}

Zongliang Liu ${ }^{\text {ał }}$, Pengfei Yu ${ }^{\text {bł }}$, Lin Dongc, Wenyan Wanga, Sijin Duan', Bingsi Wangc, Xiaoyan Gongc, Liang Ye ${ }^{b}$, Hongbo Wanga* and Jingwei Tiana ${ }^{a^{*}}$

aSchool of Pharmacy, Key Laboratory of Molecular Pharmacology and Drug Evaluation (Yantai University), Ministry of Education, Collaborative Innovation Center of Advanced Drug Delivery System and Biotech Drugs in Universities of Shandong, Yantai University, Yantai 264005, China

bDepartment of Clinical Medicine, Binzhou Medical College, Yantai 256603, China.

${ }^{\mathrm{c}}$ Luye Pharma Group, Yantai 264005, China

\section{Contents}

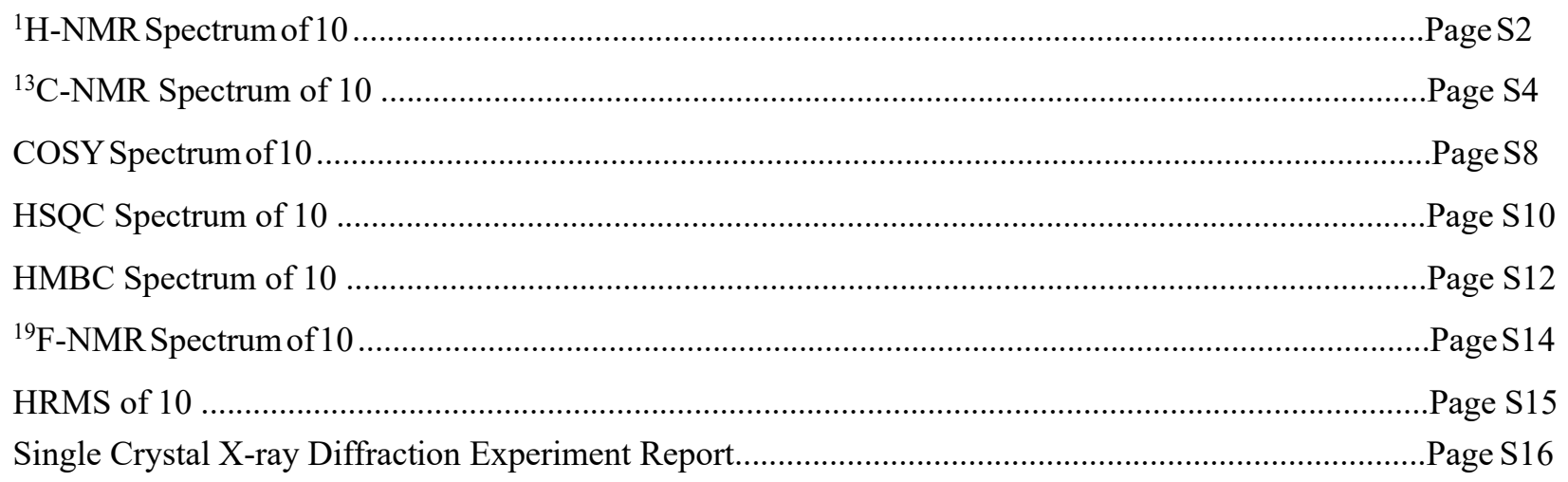




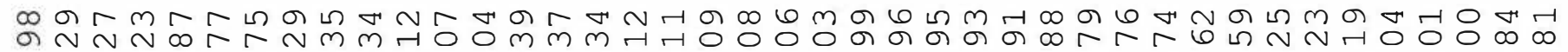
ம
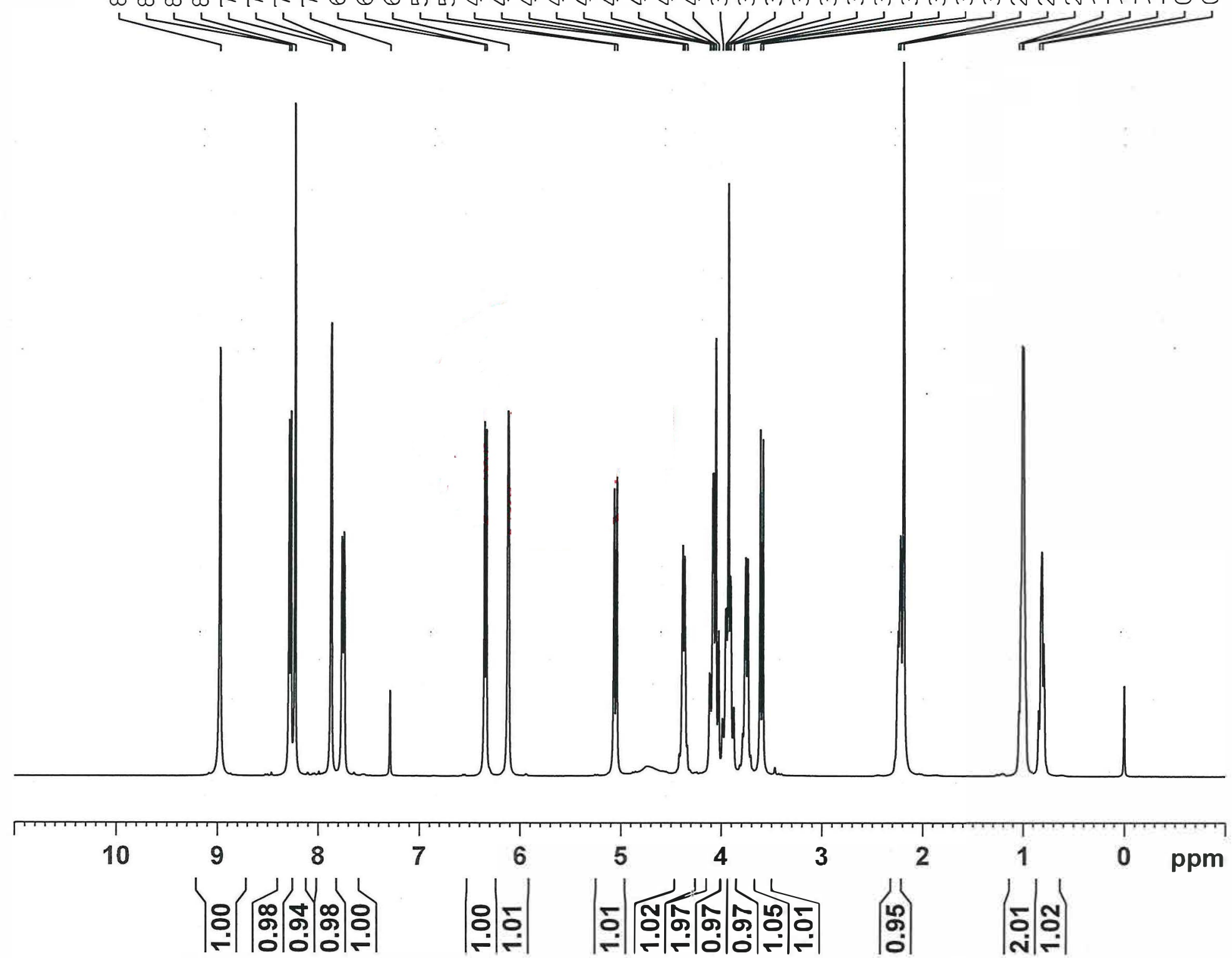

NAME

EXPNO

PROCNO

Date

Time-

INSTRUM

PROBHD

PULPROG

TD

NS

DS

SWH

AQ

RG

DW

DE

D1

TDO

SFO1

P1

SE

WDW

SSB

GB
WX-20190812-Z (FL295) 10
1 20191017 $16.29 \mathrm{~h}$ spect 0295 l 2930 65536 CDC13 16

$8012.820 \mathrm{~Hz}$ $0.244532 \mathrm{~Hz}$ 4.0894965 54.66 62.400 usec 6.50 usec $1.00000000 \mathrm{sec}$ $400.1324708 \mathrm{MHz}$

$$
\begin{array}{r}
1 \mathrm{H} \\
.00 \mathrm{usec}
\end{array}
$$
5.00 $400.1299974 \mathrm{MHz}$ $\mathrm{EM}$
0
0 $0.30 \mathrm{~Hz}$ 1.00 


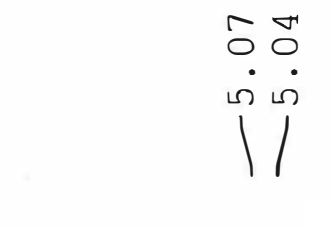

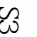

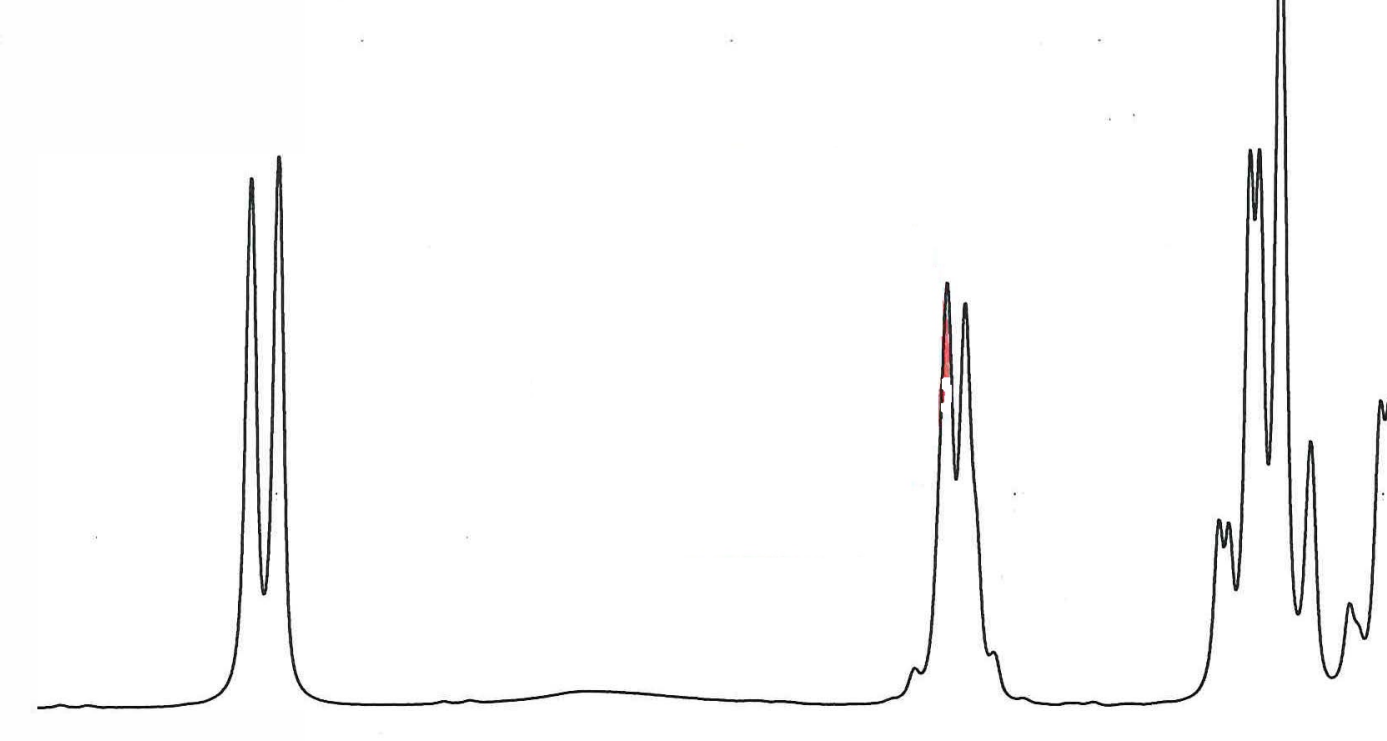

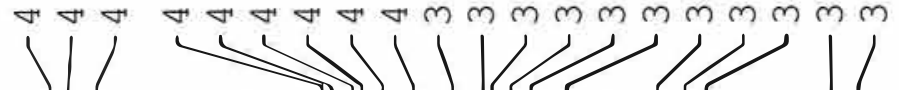

EXPNO

PROCNO

Date

Time

INSTRUM

PROBHD

PULPROG

TD

NS

DS

SWH

$A Q$

RG

$\mathrm{DE}$

TE

TDO

$\mathrm{SFO1}$

(1)

P1

SI

WDW

$S S B$

$\mathrm{LB}$
$\mathrm{GB}$
$\mathrm{PC}$
WX-20190812-Z (FL295) 10 20191017 $16.29 \mathrm{~h}$ $2116098 \quad 0295$

2930
65536 CDC13 16

$8012.820 \mathrm{~Hz}$ $0.244532 \mathrm{~Hz}$ $4.0894966 \mathrm{sec}$ 54.66 62.400 usec 6.50 usec $1.00000000 \mathrm{sec}$ 1 $\mathrm{MHz}$ $1 \mathrm{H}$ usec $400.1299974 \mathrm{MHz}$ EM $0.30 \mathrm{~Hz}$ 0.30
$\quad 0$

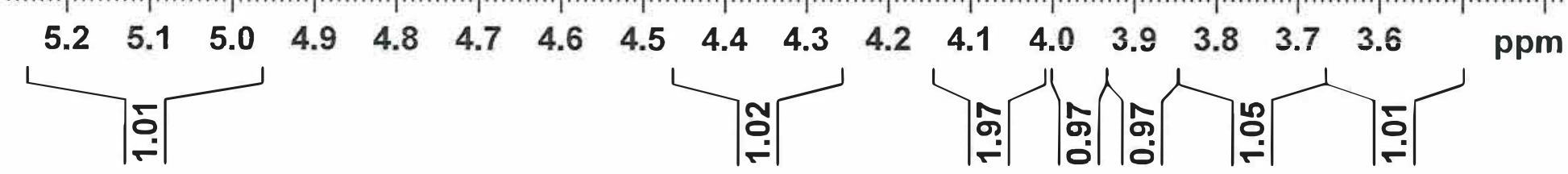

WXSH0049B样品的氢谱 $(2 / 2)$ 


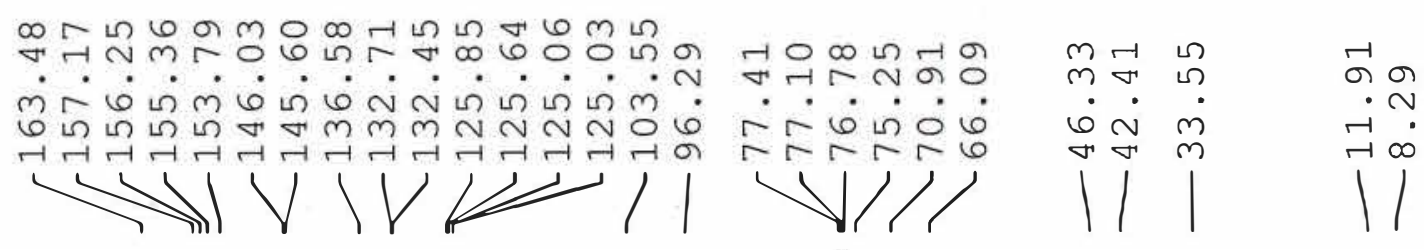

NAME

EXPNO

PROCNO

Date

INSTRUM

PROBHD

PULPROG

TD

NS

DS

SWH

AQ

RG

DW

DE

D1

D11

TDO

NEO1

$\mathrm{PI}$

SI

SF

SSB

LB

PC
WX-20190812-Z (FL295) 11 20191018 $3.50 \mathrm{~h}$ spect - 295

zgpg 30

$\mathrm{CDC} 13$

1024

$24038.461 \mathrm{~Hz}$

$0.733596 \mathrm{~Hz}$ 203.15

20.800 usec 6.50 usec
$298.0 \mathrm{~K}$

$2.00000000 \mathrm{sec}$ $0.03000000 \mathrm{sec}$ $100.6228298 \mathrm{MHz}$ $13 \mathrm{C}$ 9.50 usec 9.50
32768

$100.6127685 \mathrm{MHz}$ $\mathrm{EM}$
0 $1.00 \mathrm{~Hz}$ 0
1.40 

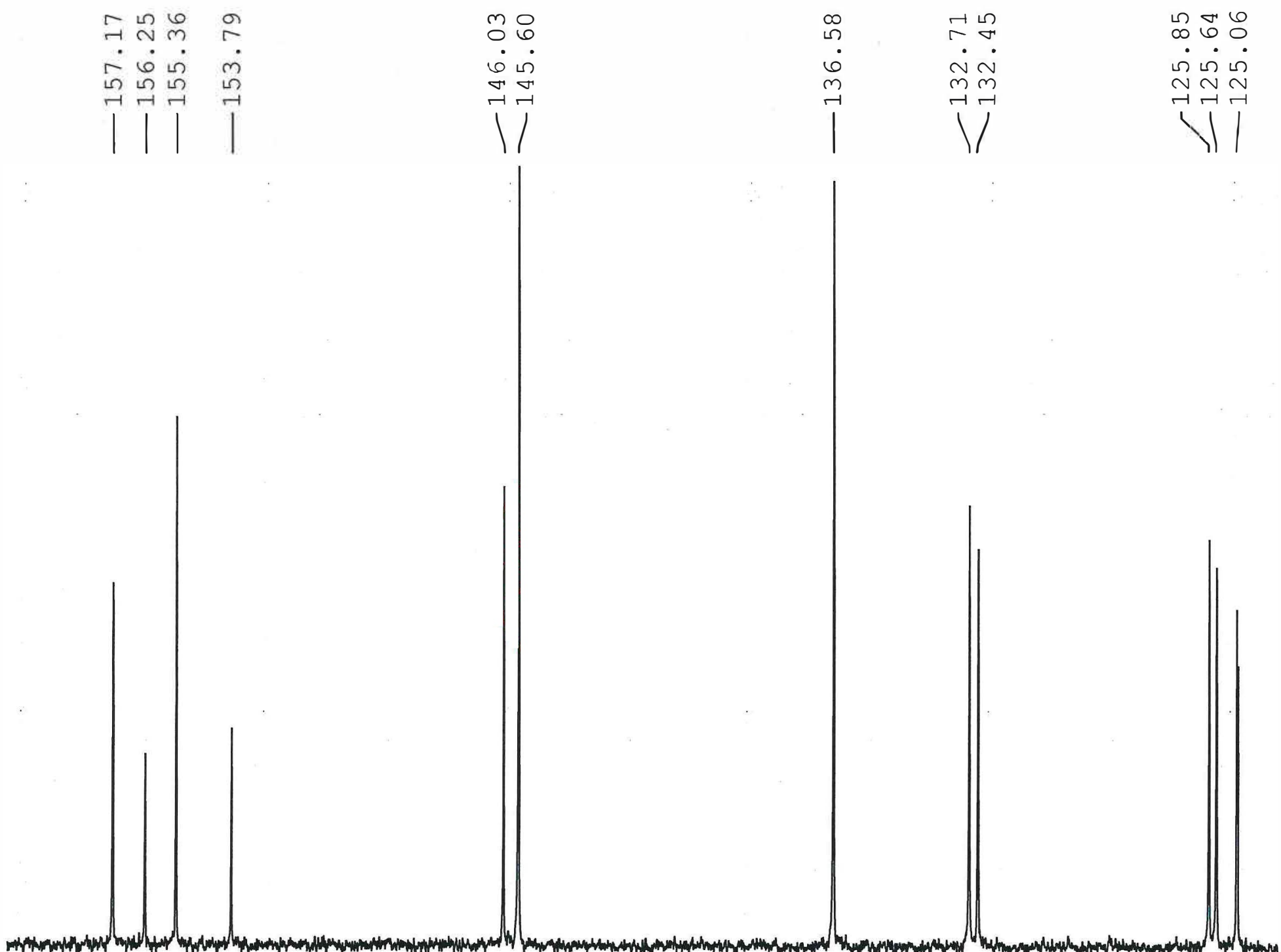

PROBHD

PULPROG

zgpg 30

65536

$\mathrm{CDCl} 3$
1024

$24038.461 \mathrm{~Hz}$

$0.733596 \mathrm{~Hz}$

203.15 20.150

usec 6.50 usec

00000000

$2.00000000 \mathrm{sec}$

1

$100.6228298 \mathrm{MHz}$

$13 \mathrm{C}$
9.50 usec

$100.6127685 \mathrm{MHz}$

EM
0
$1.00 \mathrm{~Hz}$

1.00
1.40

1.40 
WXSH0049B (TH06544-008-P1)

C13DEPT135 CDCl3 D: \\ nmr 53

요윰ำ

$\dot{0} \dot{\sim} \dot{\sim} \dot{\infty}$

तก- $\rightarrow$ -

IVV

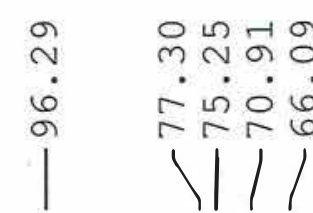

||||

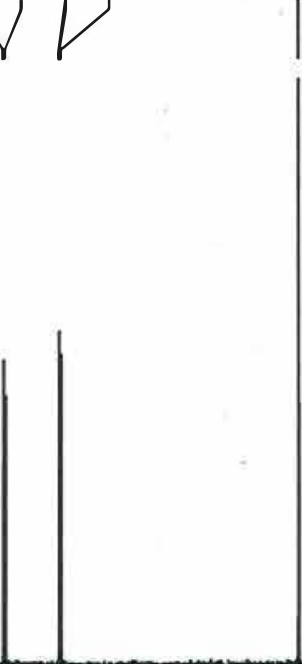

111

$m \vec{r}$

NAME

EXPNO

PROCNO

Date_

Time
INSTRUM

PROBHD

PULPROG

TD

SOLVENT

NS

DSH

SWH

$\mathrm{AQ}$

RG

DW

TE

CNST2

$\mathrm{D}$

D12

TDO

$\mathrm{SFO1}$

$\mathrm{NUCl}$

P1

SI

SF

WDW

SSB

GB

PC
WX-20190812-Z (FL295) 12 20191018 $4.20 \mathrm{~h}$ spect

eptsp135

(5) 512
512

$24038.461 \mathrm{~Hz}$

$0.733596 \mathrm{~Hz}$$$
203.15
$$

20.800 usec

6.50 usec $298.0 \mathrm{~K}$

145.0000000

$2.00000000 \mathrm{sec}$

$0.00344828 \mathrm{sec}$

0.00002000 sec

$100.6208175 \mathrm{MHz}$ $13 \mathrm{C}$ $13 \mathrm{C}$ 2000.00 usec $00.6127685 \mathrm{MHz}$

EM $1.00 \mathrm{~Hz}$ 1.40 

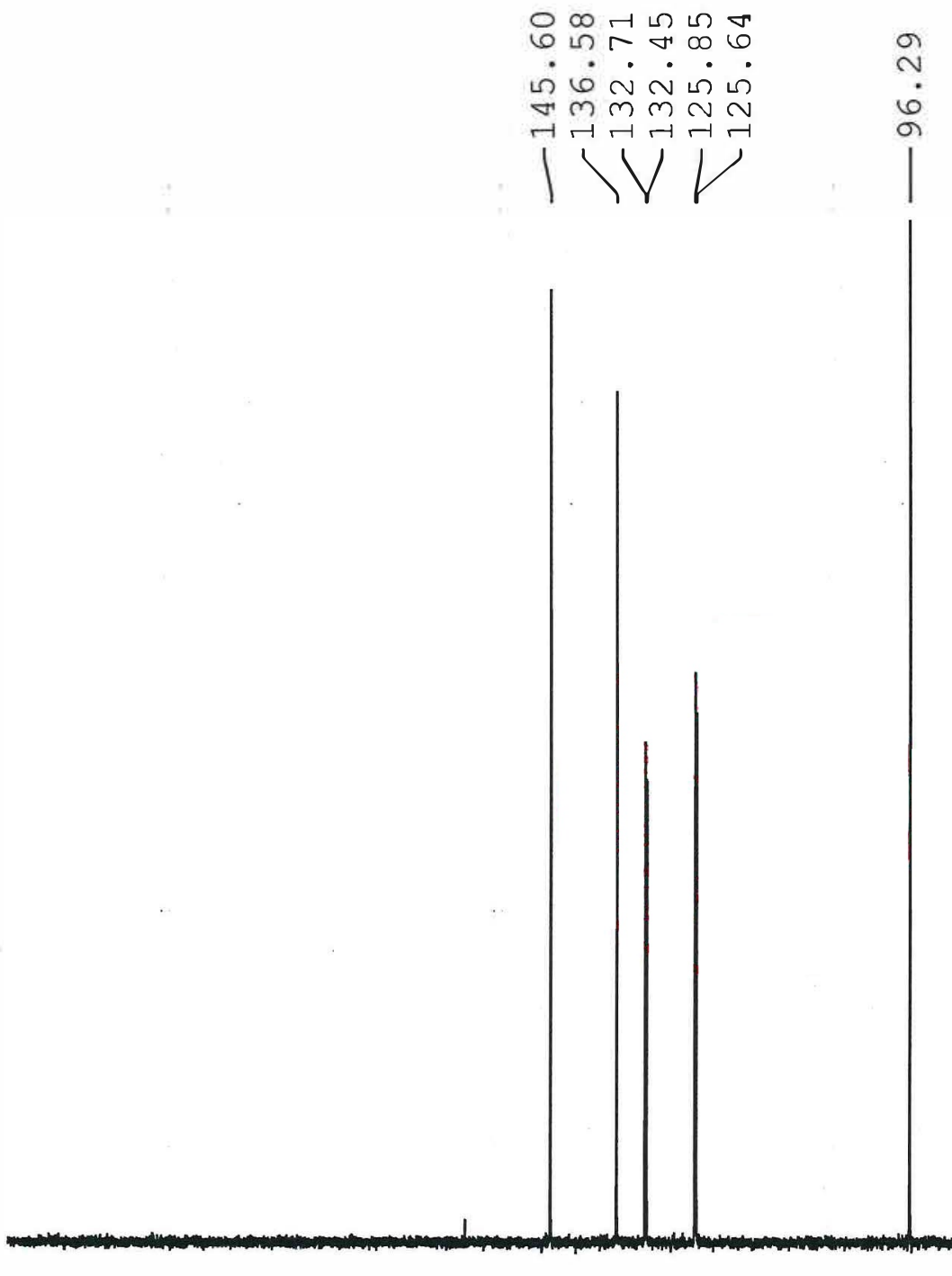

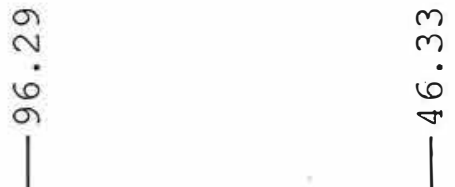

m

AME

EXPNO

PROCNO

Date

INSTRUM

PROBHD

PULPROG

TD

NS

DS

SWH

FIDRES

AQ

RG

DW

TE

CNST2

D1

D2

D12

FO1

NUC1

P1

P13

SI

WDW

SSB

LB

GB
WX-20190812-Z (FL295) 20191018 5.30 5.30
spect 21160980295 deptsp90 65536 512

$24038.461 \mathrm{~Hz}$ $0.733596 \mathrm{~Hz}$ $1.3631988 \mathrm{sec}$ 203.15 20.800 usec 6.50 usec 45.0000000 $2.00000000 \mathrm{sec}$ $0.00344828 \mathrm{sec}$ $0.00002000 \mathrm{sec}$

$100.6228298 \mathrm{MHz}$ $13 \mathrm{C}$

9.50 use 2000.00 use 100.6127685 MH EM
0
$1.00 \mathrm{~Hz}$
0
1.40

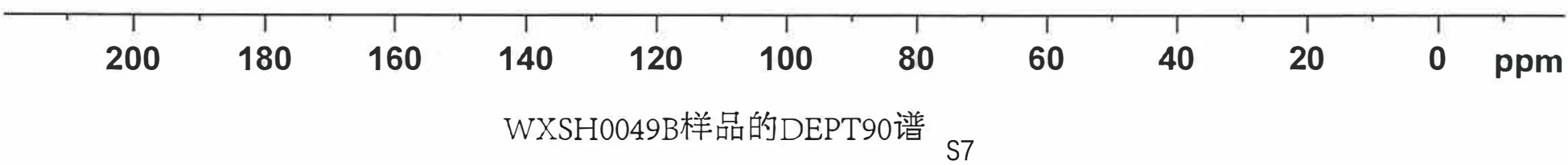




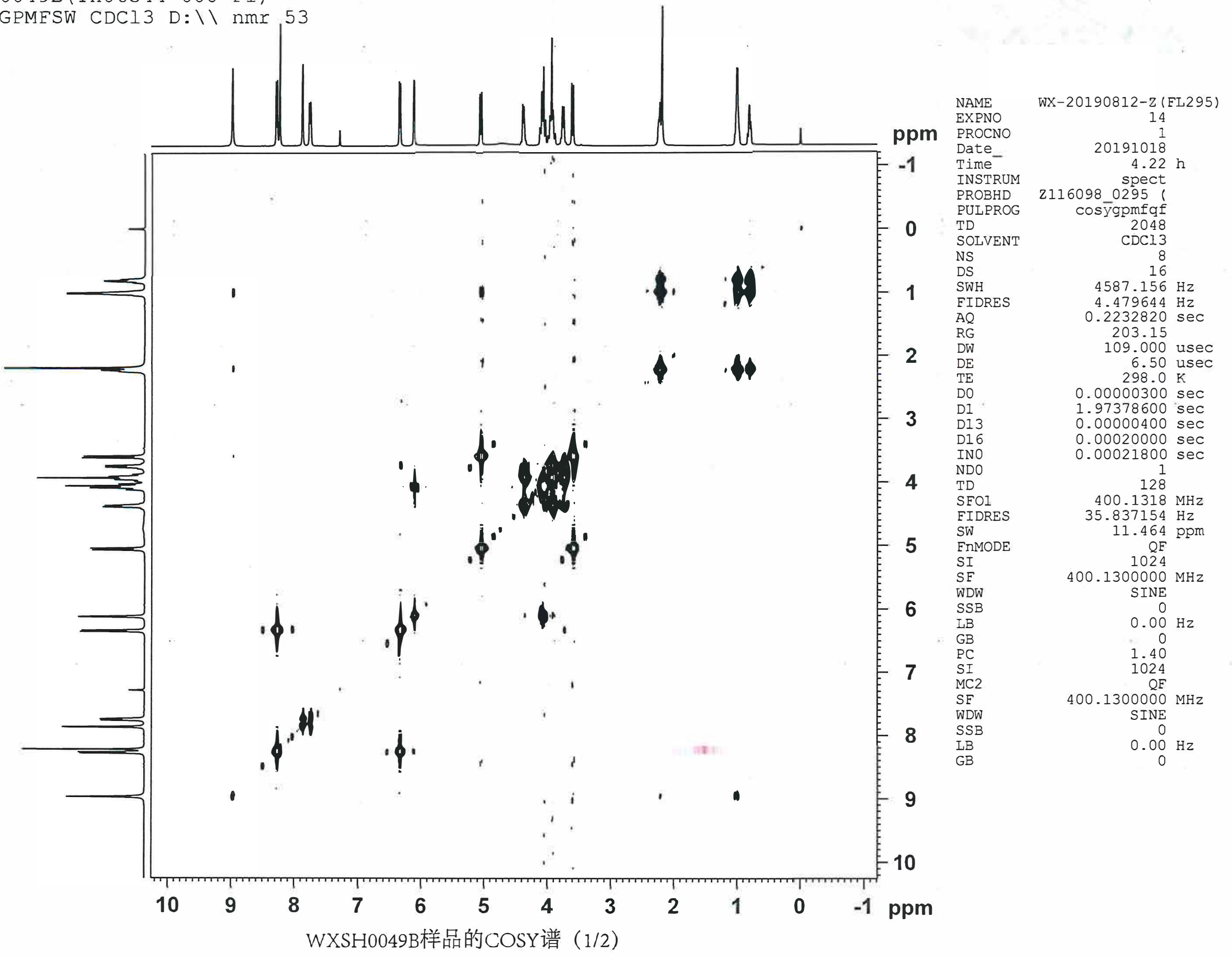




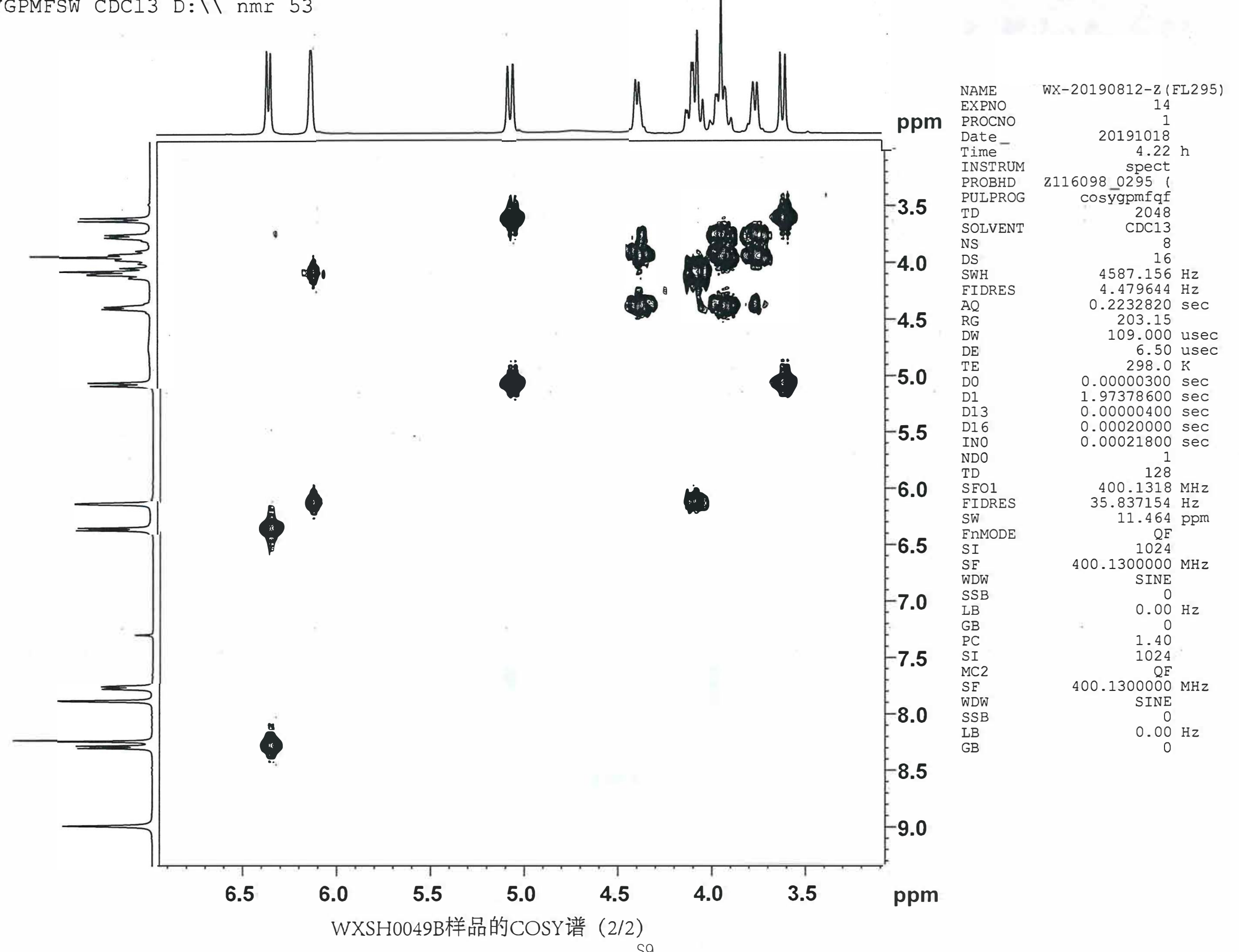




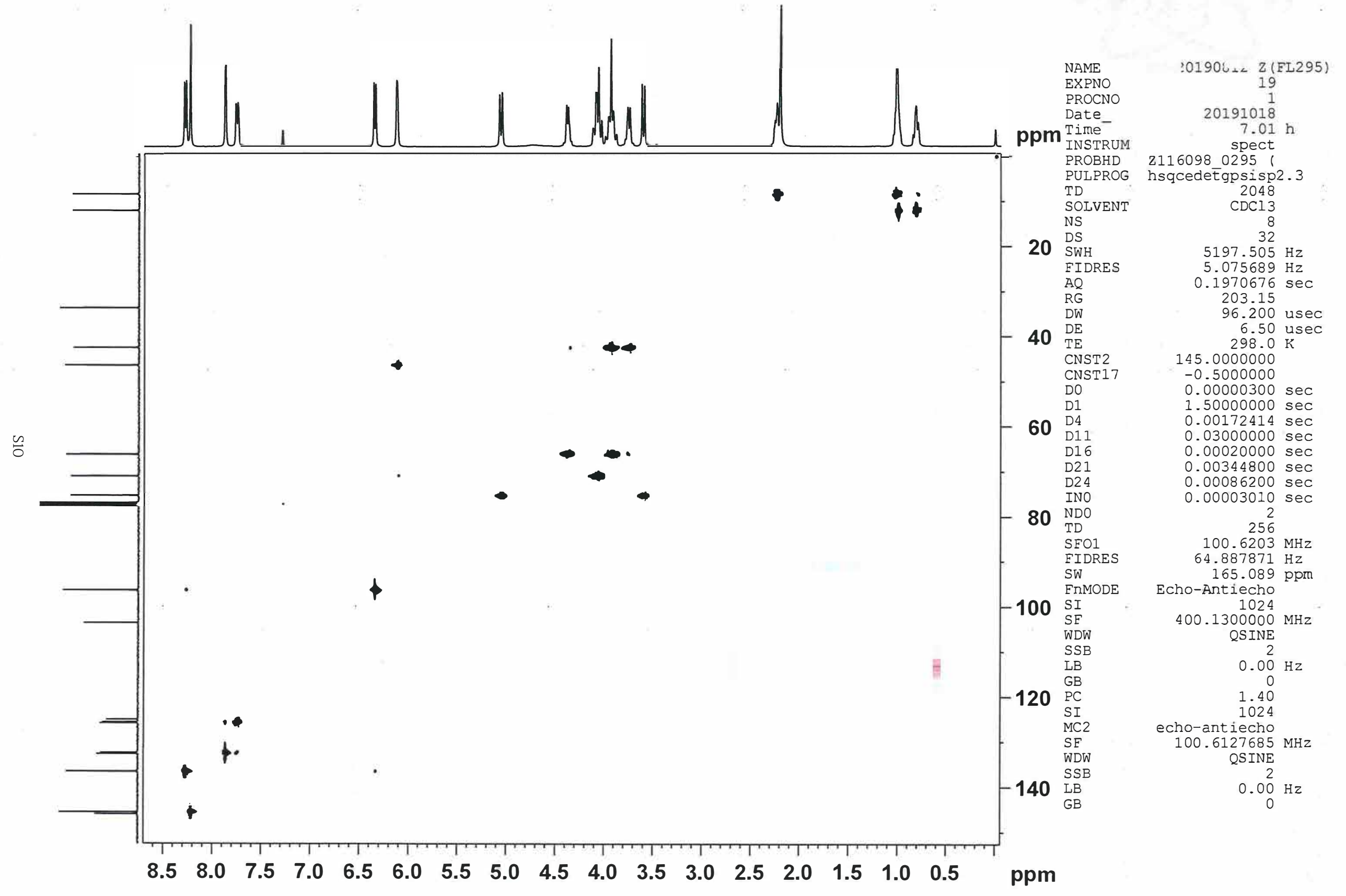




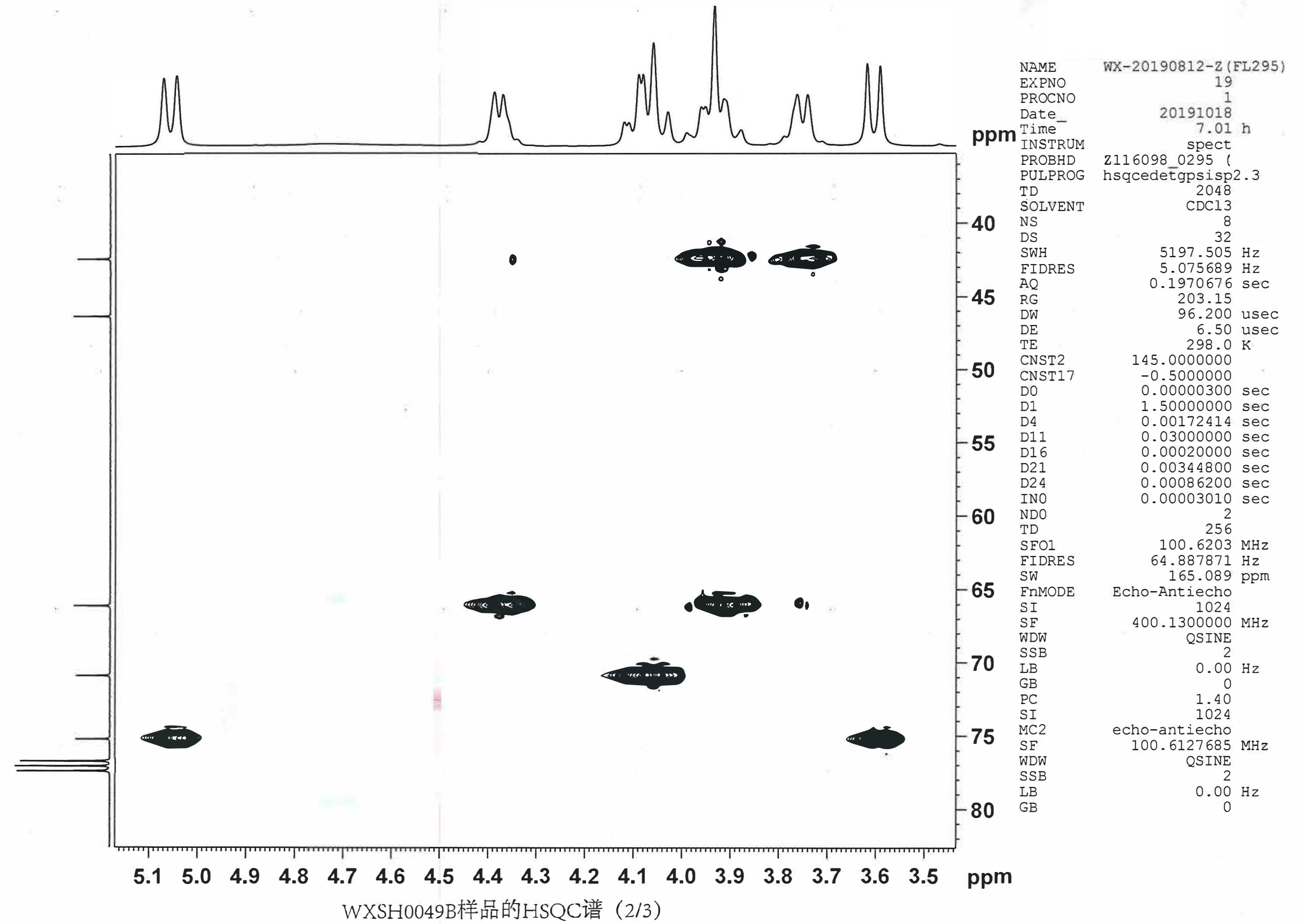


WXSH0 049B (TH06544-008-P1)

HMBCETGPL3ND CDC13 D: \\ nmr 53

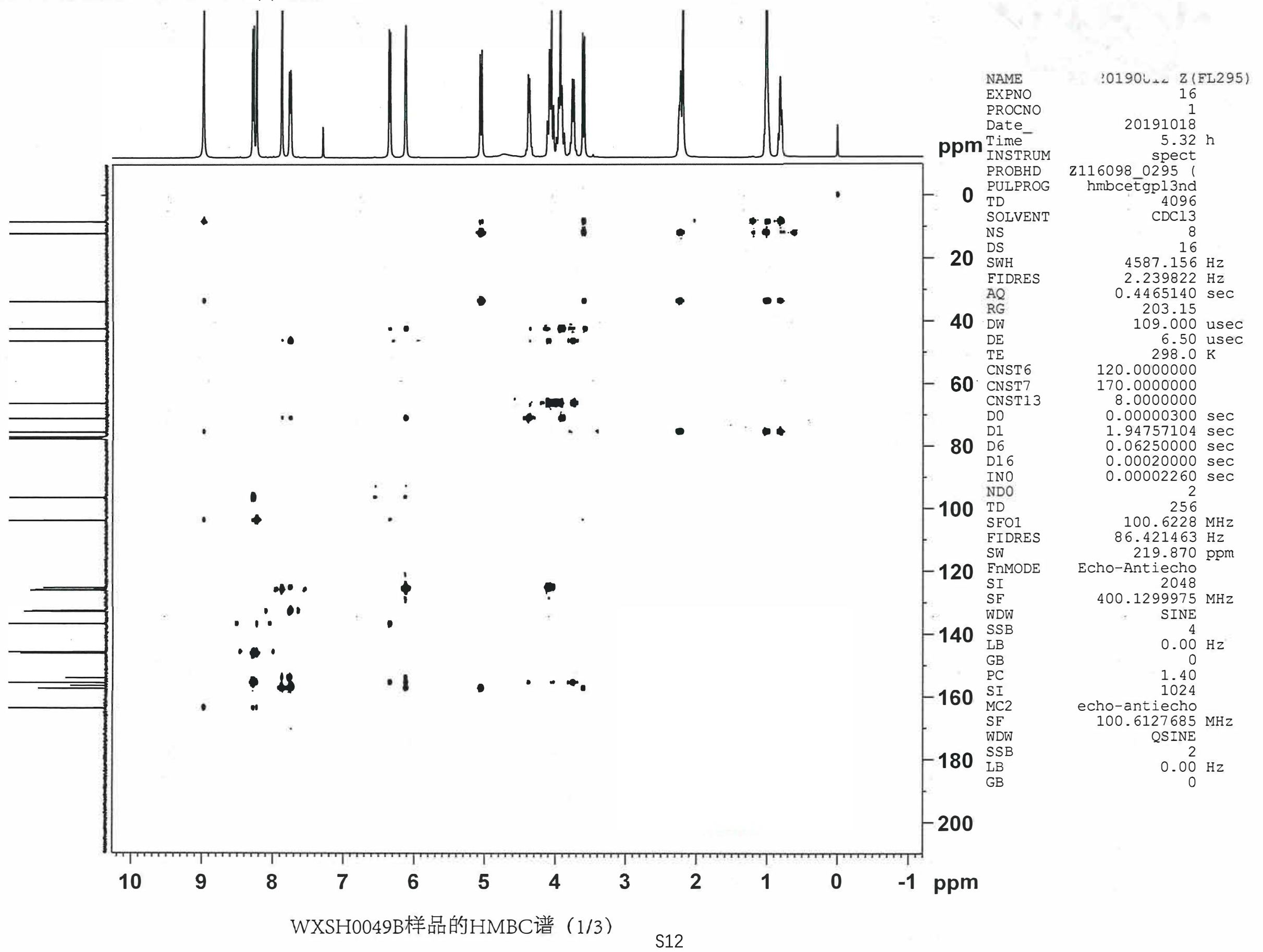




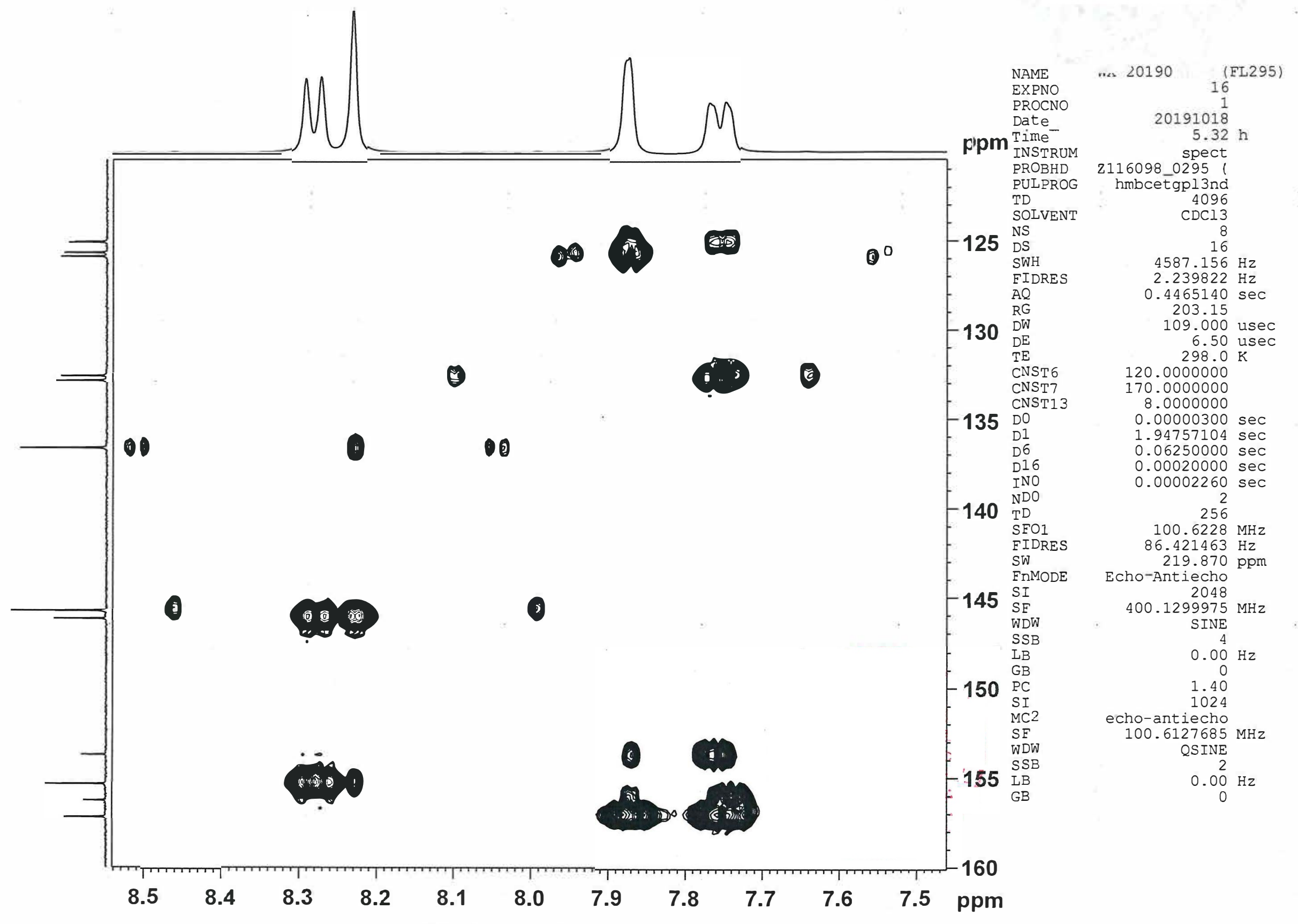

WXSH0049B样品的HMBC谱 $(2 / 3)$ 


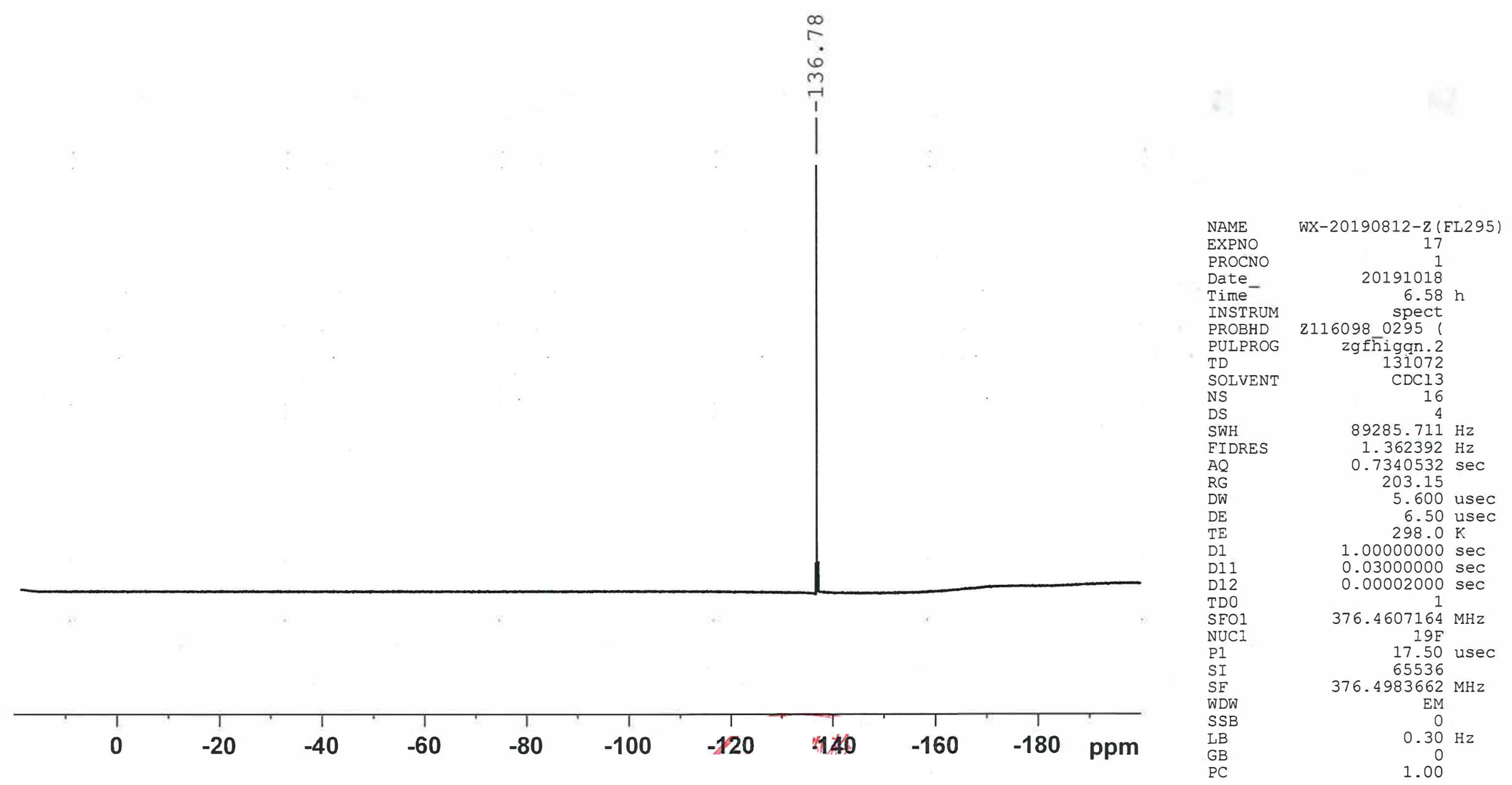

WXSH0049B样品的19F谱 
IRM Calibration Status 1

InjPosition

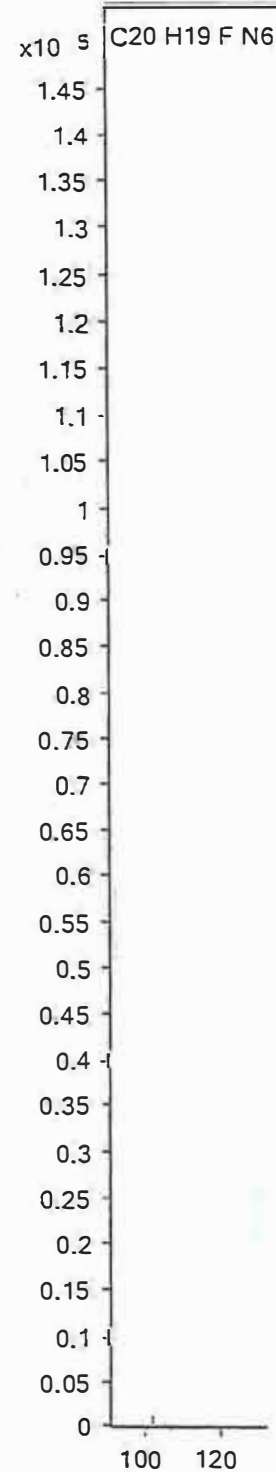

174 min, 3 scans) WXSH0049B-T.H06544-008-P1.d Subtract

Acquired Time

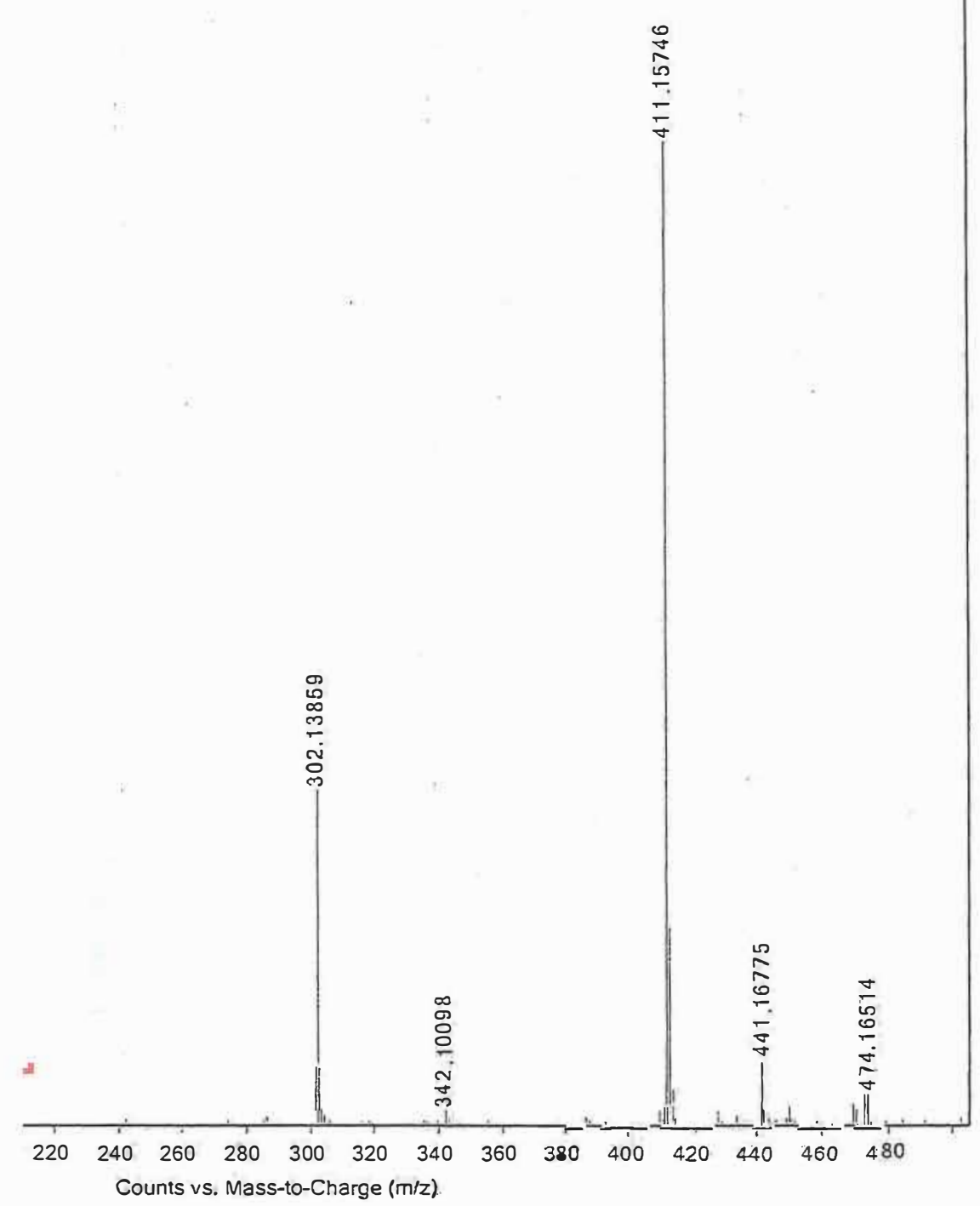

WXSH0049B样品的高分辨质谱图 (正离子) 


\section{Single Crystal X-ray Diffraction Experiment Report}

Sample Name: LPM4870108 

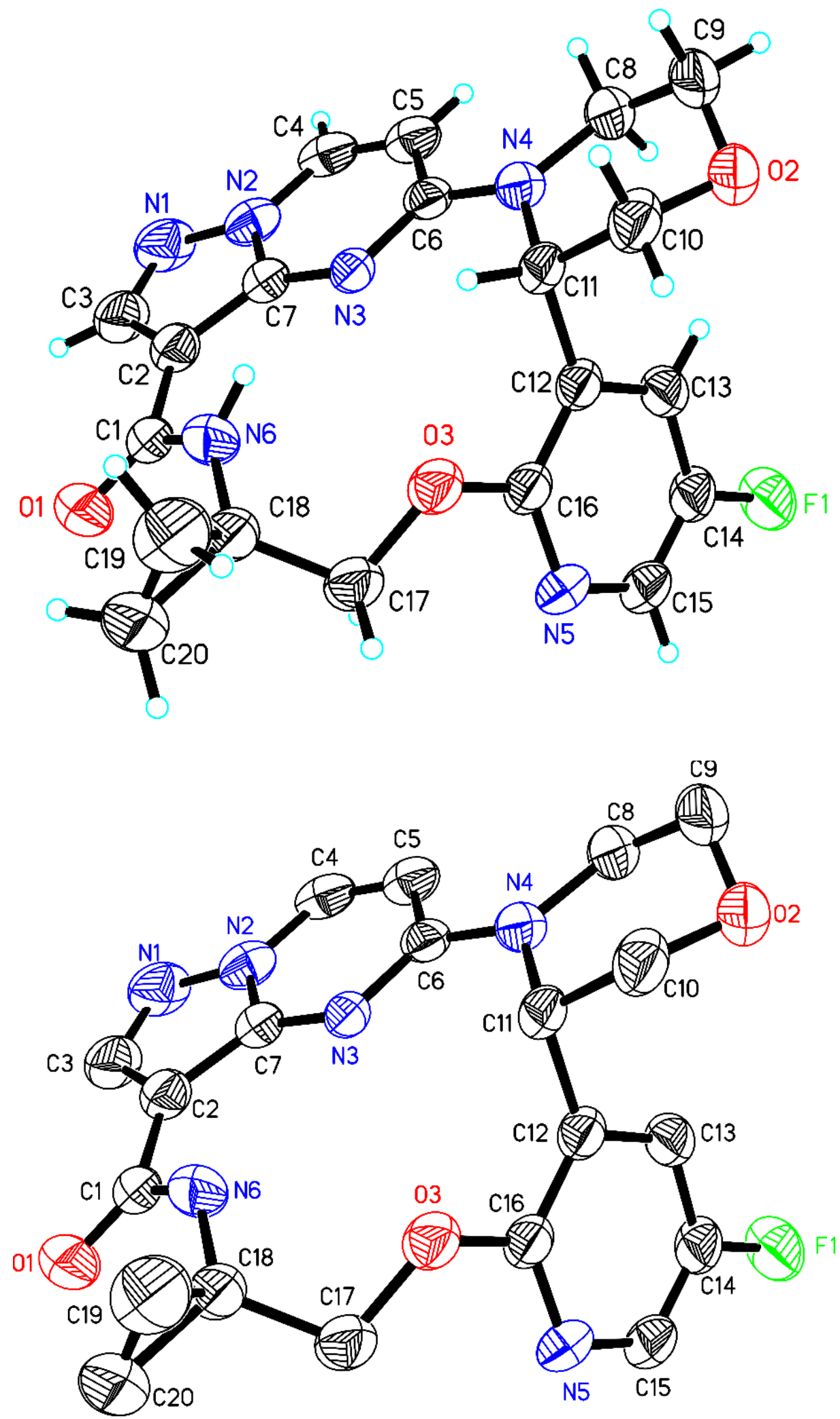

LPM4870108 Three-dimensional structure ellipsoid diagram 


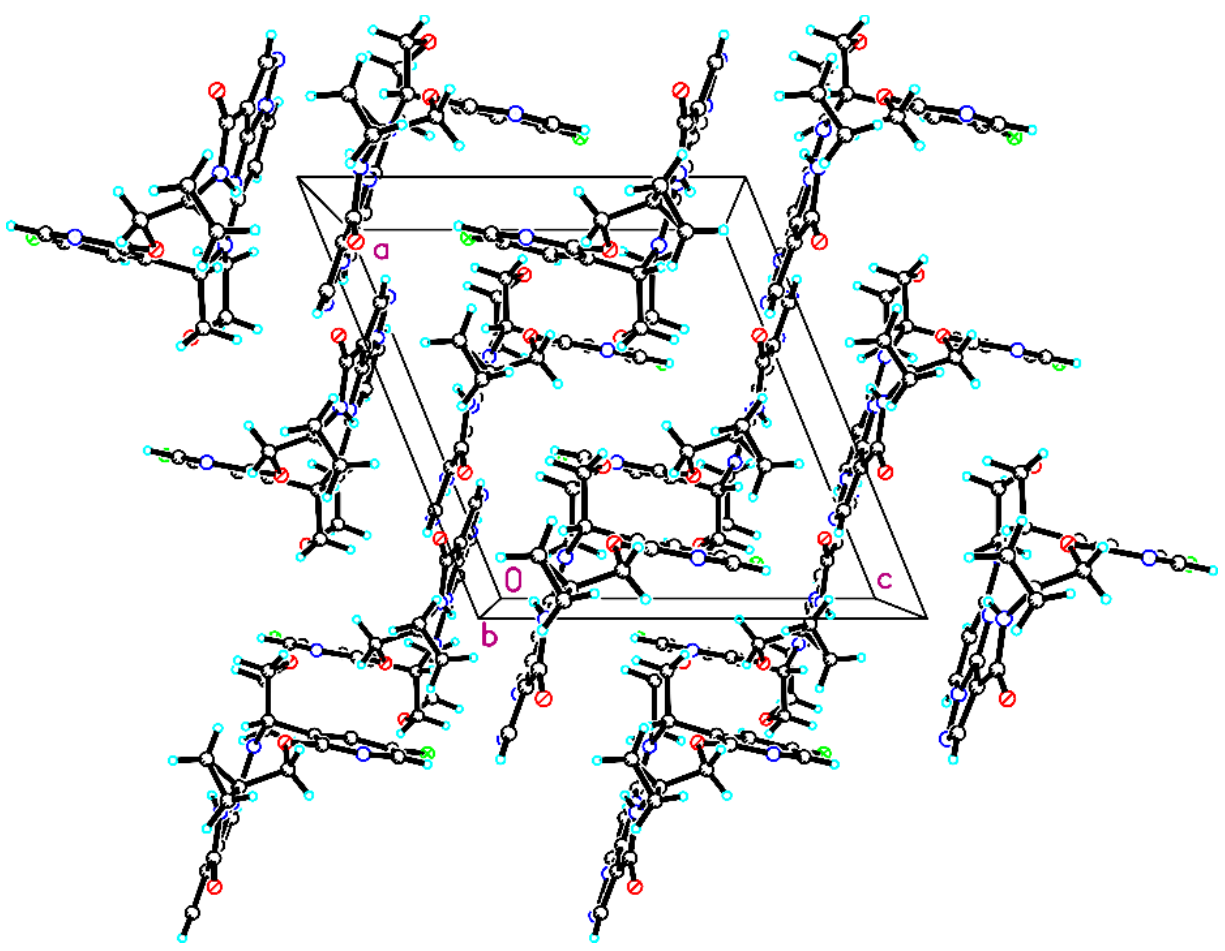

LPM4870108 unit cell stacking diagram along the $\mathrm{b}$ axis<smiles>O=C(NC1(COc2ccc(F)cn2)CC1)c1cnn2ccc(N3CCOCC3)nc12</smiles>

LPM4870108 absolute configuration 
Table 1. Crystal data and structure refinement for LPM4870108.

\begin{tabular}{|c|c|}
\hline Identification code & LPM4870108 \\
\hline Empirical formula & C20 H19 F N6 03 \\
\hline Formula weight & 410.41 \\
\hline Temperature & $296(2) \mathrm{K}$ \\
\hline Wavelength & $1.54178 \mathrm{~A}$ \\
\hline Crystal system, space group & Monoclinic, $\quad \mathrm{C} 2$ \\
\hline Unit cell dimensions & $\begin{array}{l}\mathrm{a}=12.757(5) \text { A } \quad \text { alpha }=90 \text { deg. } \\
\mathrm{b}=16.289(6) \mathrm{A} \quad \text { beta }=112.330(16) \mathrm{deg} . \\
\mathrm{c}=11.971(7) \mathrm{A} \quad \text { gamma }=90 \mathrm{deg} .\end{array}$ \\
\hline Volume & $2301.0(19) \quad A^{\wedge} 3$ \\
\hline Z, Calculated density & $4, \quad 1.185 \mathrm{Mg} / \mathrm{m}^{\wedge} 3$ \\
\hline Absorption coefficient & $0.740 \mathrm{~mm}^{\wedge}-1$ \\
\hline $\mathrm{F}(000)$ & 856 \\
\hline Crystal size & $0.24 \times 0.10 \times 0.08 \mathrm{~mm}$ \\
\hline Theta range for data collection & 3. 992 to 68.739 deg. \\
\hline Limiting indices & $-15<=\mathrm{h}<=15, \quad-19<=\mathrm{k}<=19, \quad-14<=1<=13$ \\
\hline Reflections collected / unique & $14159 / 4157[\mathrm{R}(\mathrm{int})=0.0815]$ \\
\hline Completeness to theta $=67.679$ & $99.9 \%$ \\
\hline Absorption correction & None \\
\hline Refinement method & Ful1-matrix least-squares on $\mathrm{F}^{\wedge} 2$ \\
\hline Data / restraints / parameters & $4157 / 1 / 272$ \\
\hline Goodness-of-fit on $\mathrm{F}^{\wedge} 2$ & 1.117 \\
\hline Final $R$ indices $[\mathrm{I}>2 \operatorname{sigma}(\mathrm{I})]$ & $\mathrm{R} 1=0.0623, \quad$ wR2 $=0.1815$ \\
\hline $\mathrm{R}$ indices (all data) & $\mathrm{R} 1=0.0869, \quad \mathrm{wR} 2=0.2016$ \\
\hline Absolute structure parameter & $-0.02(12)$ \\
\hline Extinction coefficient & $0.0013(4)$ \\
\hline Largest diff. peak and hole & 0.267 and -0.226 e. $A^{\wedge}-3$ \\
\hline
\end{tabular}


Table 2. Atomic coordinates ( x 10^4) and equivalent isotropic displacement parameters (A^2 x 10^3) for LPM4870108.

\begin{tabular}{lllrr}
\hline & $\mathrm{x}$ & $\mathrm{y}$ & $\mathrm{z}$ & $\mathrm{U}(\mathrm{eq})$ \\
& & & & \\
\hline $\mathrm{F}(1)$ & $3825(4)$ & $3389(3)$ & $3235(4)$ & $110(1)$ \\
$\mathrm{O}(1)$ & $6651(4)$ & $6701(3)$ & $9172(4)$ & $107(2)$ \\
0(2) & $1510(4)$ & $2932(3)$ & $5823(4)$ & $93(1)$ \\
0(3) & $3292(3)$ & $5638(2)$ & $6281(3)$ & $74(1)$ \\
$\mathrm{N}(1)$ & $7794(4)$ & $4332(4)$ & $10581(5)$ & $98(2)$ \\
$\mathrm{N}(2)$ & $6795(3)$ & $3982(3)$ & $9862(4)$ & $73(1)$ \\
$\mathrm{N}(3)$ & $4953(3)$ & $4334(2)$ & $8460(3)$ & $56(1)$ \\
$\mathrm{N}(4)$ & $3645(4)$ & $3334(3)$ & $7554(4)$ & $67(1)$ \\
$\mathrm{N}(5)$ & $3611(4)$ & $5320(3)$ & $4553(4)$ & $74(1)$ \\
$\mathrm{N}(6)$ & $4932(4)$ & $6113(3)$ & $8444(4)$ & $78(1)$ \\
$\mathrm{C}(1)$ & $6060(5)$ & $6111(3)$ & $9052(4)$ & $70(1)$ \\
$\mathrm{C}(2)$ & $6508(4)$ & $5307(4)$ & $9547(5)$ & $71(1)$ \\
$\mathrm{C}(3)$ & $7584(5)$ & $5109(5)$ & $10373(5)$ & $94(2)$ \\
$\mathrm{C}(4)$ & $6554(6)$ & $3159(4)$ & $9688(6)$ & $77(2)$ \\
$\mathrm{C}(5)$ & $5541(5)$ & $2924(3)$ & $8947(5)$ & $74(1)$ \\
$\mathrm{C}(6)$ & $4704(5)$ & $3537(3)$ & $8312(4)$ & $60(1)$ \\
$\mathrm{C}(7)$ & $5994(4)$ & $4537(3)$ & $9212(4)$ & $59(1)$ \\
$\mathrm{C}(8)$ & $3309(7)$ & $2478(4)$ & $7281(6)$ & $88(2)$ \\
$\mathrm{C}(9)$ & $2040(7)$ & $2374(5)$ & $6757(7)$ & $105(3)$ \\
$\mathrm{C}(10)$ & $1678(5)$ & $3732(5)$ & $6327(5)$ & $82(2)$ \\
$\mathrm{C}(11)$ & $2926(4)$ & $3984(3)$ & $6803(4)$ & $61(1)$ \\
$\mathrm{C}(12)$ & $3262(4)$ & $4237(3)$ & $5747(4)$ & $61(1)$ \\
$\mathrm{C}(13)$ & $3395(5)$ & $3664(4)$ & $4955(5)$ & $72(1)$ \\
$\mathrm{C}(14)$ & $3653(5)$ & $3949(4)$ & $3999(5)$ & $76(1)$ \\
$\mathrm{C}(15)$ & $3748(5)$ & $4743(4)$ & $3810(5)$ & $79(2)$ \\
$\mathrm{C}(16)$ & $3404(4)$ & $5062(3)$ & $5509(4)$ & $63(1)$ \\
$\mathrm{C}(17)$ & $3854(7)$ & $6413(4)$ & $6334(5)$ & $86(2)$ \\
$\mathrm{C}(18)$ & $4244(6)$ & $6705(3)$ & $7617(5)$ & $78(2)$ \\
$\mathrm{C}(19)$ & $3507(10)$ & $7230(5)$ & $7992(9)$ & $121(3)$ \\
$\mathrm{C}(20)$ & $4452(9)$ & $7593(4)$ & $7820(8)$ & $110(2)$ \\
$\mathrm{H}(6 \mathrm{~A})$ & 4577 & 5698 & 8573 & 94 \\
$\mathrm{H}(3 \mathrm{~B})$ & 8121 & 5509 & 10753 & 113 \\
$\mathrm{H}(4 \mathrm{~A})$ & 7102 & 2772 & 10091 & 92 \\
$\mathrm{H}(5 \mathrm{~B})$ & 5365 & 2368 & 8831 & 89 \\
$\mathrm{H}(8 \mathrm{~A})$ & 3625 & 2156 & 8016 & 106 \\
$\mathrm{H}(8 \mathrm{~B})$ & 3619 & 2269 & 6712 & 106 \\
$\mathrm{H}(9 \mathrm{~A})$ & 1857 & 1819 & 6450 & 127 \\
$\mathrm{H}(9 \mathrm{~B})$ & 1750 & 2451 & 7390 & 127 \\
$\mathrm{H}(10 \mathrm{~A})$ & 1409 & 3749 & 6983 & 99 \\
& & & &
\end{tabular}




\begin{tabular}{llllr} 
H(10B) & 1236 & 4121 & 5717 & 99 \\
H(11A) & 3011 & 4467 & 7317 & 73 \\
H(13A) & 3314 & 3105 & 5063 & 86 \\
H(15A) & 3912 & 4907 & 3150 & 95 \\
H(17A) & 3336 & 6807 & 5795 & 103 \\
H(17B) & 4496 & 6345 & 6096 & 103 \\
H(19A) & 2757 & 7350 & 7399 & 145 \\
H(19B) & 3547 & 7173 & 8814 & 145 \\
H(20A) & 5090 & 7764 & 8533 & 133 \\
H(20B) & 4299 & 7940 & 7118 & 133 \\
& & & & \\
\hline
\end{tabular}


Table 3. Bond lengths [A] and angles [deg] for LPM4870108.

\begin{tabular}{|c|c|c|c|}
\hline $\mathrm{F}(1)-\mathrm{C}(14)$ & 1. $368(6)$ & $\mathrm{C}(5)-\mathrm{H}(5 \mathrm{~B})$ & 0.9300 \\
\hline $0(1)-C(1)$ & $1.196(7)$ & $C(8)-C(9)$ & $1.506(11)$ \\
\hline $0(2)-C(9)$ & 1. $400(10)$ & $\mathrm{C}(8)-\mathrm{H}(8 \mathrm{~A})$ & 0.9700 \\
\hline $0(2)-C(10)$ & $1.417(8)$ & $\mathrm{C}(8)-\mathrm{H}(8 \mathrm{~B})$ & 0.9700 \\
\hline $0(3)-C(16)$ & 1. $363(6)$ & $\mathrm{C}(9)-\mathrm{H}(9 \mathrm{~A})$ & 0.9700 \\
\hline $0(3)-C(17)$ & 1. $440(8)$ & $\mathrm{C}(9)-\mathrm{H}(9 \mathrm{~B})$ & 0.9700 \\
\hline$N(1)-C(3)$ & 1. $297(10)$ & $C(10)-C(11)$ & 1. $529(7)$ \\
\hline$N(1)-N(2)$ & $1.362(7)$ & $\mathrm{C}(10)-\mathrm{H}(10 \mathrm{~A})$ & 0.9700 \\
\hline $\mathrm{N}(2)-\mathrm{C}(7)$ & 1. $365(6)$ & $\mathrm{C}(10)-\mathrm{H}(10 \mathrm{~B})$ & 0.9700 \\
\hline$N(2)-C(4)$ & 1. $373(8)$ & $C(11)-C(12)$ & 1. $538(7)$ \\
\hline$N(3)-C(6)$ & 1. $331(6)$ & $\mathrm{C}(11)-\mathrm{H}(11 \mathrm{~A})$ & 0.9800 \\
\hline$N(3)-C(7)$ & 1. $332(6)$ & $C(12)-C(13)$ & 1. $386(7)$ \\
\hline $\mathrm{N}(4)-\mathrm{C}(6)$ & 1. $352(7)$ & $C(12)-C(16)$ & 1. $399(7)$ \\
\hline$N(4)-C(8)$ & 1. $460(7)$ & $C(13)-C(14)$ & 1. $386(7)$ \\
\hline$N(4)-C(11)$ & $1.463(7)$ & $\mathrm{C}(13)-\mathrm{H}(13 \mathrm{~A})$ & 0.9300 \\
\hline$N(5)-C(16)$ & 1. $336(6)$ & $C(14)-C(15)$ & 1. $327(9)$ \\
\hline$N(5)-C(15)$ & 1. $350(8)$ & $C(15)-H(15 A)$ & 0.9300 \\
\hline$N(6)-C(1)$ & 1. $343(7)$ & $C(17)-C(18)$ & $1.501(8)$ \\
\hline$N(6)-C(18)$ & 1. 421(7) & $\mathrm{C}(17)-\mathrm{H}(17 \mathrm{~A})$ & 0.9700 \\
\hline $\mathrm{N}(6)-\mathrm{H}(6 \mathrm{~A})$ & 0.8600 & $\mathrm{C}(17)-\mathrm{H}(17 \mathrm{~B})$ & 0.9700 \\
\hline$C(1)-C(2)$ & $1.460(8)$ & $C(18)-C(19)$ & 1. $462(10)$ \\
\hline$C(2)-C(3)$ & 1. $391(8)$ & $C(18)-C(20)$ & 1. $473(9)$ \\
\hline$C(2)-C(7)$ & 1. $402(8)$ & $C(19)-C(20)$ & 1. $426(14)$ \\
\hline $\mathrm{C}(3)-\mathrm{H}(3 \mathrm{~B})$ & 0.9300 & $\mathrm{C}(19)-\mathrm{H}(19 \mathrm{~A})$ & 0.9700 \\
\hline$C(4)-C(5)$ & 1. $316(9)$ & $\mathrm{C}(19)-\mathrm{H}(19 \mathrm{~B})$ & 0.9700 \\
\hline $\mathrm{C}(4)-\mathrm{H}(4 \mathrm{~A})$ & 0.9300 & $\mathrm{C}(20)-\mathrm{H}(20 \mathrm{~A})$ & 0.9700 \\
\hline$C(5)-C(6)$ & 1. $449(7)$ & $\mathrm{C}(20)-\mathrm{H}(20 \mathrm{~B})$ & 0.9700 \\
\hline$C(9)-0(2)-C(10)$ & $107.9(5)$ & $0(1)-\mathrm{C}(1)-\mathrm{N}(6)$ & $124.0(6)$ \\
\hline$C(16)-0(3)-C(17)$ & $117.1(4)$ & $0(1)-C(1)-C(2)$ & $122.6(5)$ \\
\hline $\mathrm{C}(3)-\mathrm{N}(1)-\mathrm{N}(2)$ & $102.2(5)$ & $N(6)-C(1)-C(2)$ & 113. 4(4) \\
\hline$N(1)-N(2)-C(7)$ & $113.7(5)$ & $C(3)-C(2)-C(7)$ & $102.9(5)$ \\
\hline$N(1)-N(2)-C(4)$ & $127.3(5)$ & $C(3)-C(2)-C(1)$ & $128.7(5)$ \\
\hline $\mathrm{C}(7)-\mathrm{N}(2)-\mathrm{C}(4)$ & $118.9(5)$ & $C(7)-C(2)-C(1)$ & $128.1(4)$ \\
\hline$C(6)-N(3)-C(7)$ & $117.3(4)$ & $\mathrm{N}(1)-\mathrm{C}(3)-\mathrm{C}(2)$ & 116. $0(6)$ \\
\hline $\mathrm{C}(6)-\mathrm{N}(4)-\mathrm{C}(8)$ & $121.2(5)$ & $\mathrm{N}(1)-\mathrm{C}(3)-\mathrm{H}(3 \mathrm{~B})$ & 122.0 \\
\hline $\mathrm{C}(6)-\mathrm{N}(4)-\mathrm{C}(11)$ & $118.0(4)$ & $\mathrm{C}(2)-\mathrm{C}(3)-\mathrm{H}(3 \mathrm{~B})$ & 122.0 \\
\hline $\mathrm{C}(8)-\mathrm{N}(4)-\mathrm{C}(11)$ & $119.5(4)$ & $\mathrm{C}(5)-\mathrm{C}(4)-\mathrm{N}(2)$ & $119.5(5)$ \\
\hline $\mathrm{C}(16)-\mathrm{N}(5)-\mathrm{C}(15)$ & $117.5(5)$ & $\mathrm{C}(5)-\mathrm{C}(4)-\mathrm{H}(4 \mathrm{~A})$ & 120.2 \\
\hline $\mathrm{C}(1)-\mathrm{N}(6)-\mathrm{C}(18)$ & $129.1(5)$ & $\mathrm{N}(2)-\mathrm{C}(4)-\mathrm{H}(4 \mathrm{~A})$ & 120.2 \\
\hline $\mathrm{C}(1)-\mathrm{N}(6)-\mathrm{H}(6 \mathrm{~A})$ & 115.5 & $C(4)-C(5)-C(6)$ & $119.4(5)$ \\
\hline $\mathrm{C}(18)-\mathrm{N}(6)-\mathrm{H}(6 \mathrm{~A})$ & 115.5 & $\mathrm{C}(4)-\mathrm{C}(5)-\mathrm{H}(5 \mathrm{~B})$ & 120.3 \\
\hline
\end{tabular}




\begin{tabular}{|c|c|c|c|}
\hline$C(6)-C(5)-H(5 B)$ & 120.3 & $\mathrm{C}(14)-\mathrm{C}(13)-\mathrm{H}(13 \mathrm{~A})$ & 121.1 \\
\hline $\mathrm{N}(3)-\mathrm{C}(6)-\mathrm{N}(4)$ & $117.1(4)$ & $C(12)-C(13)-H(13 A)$ & 121.1 \\
\hline$N(3)-C(6)-C(5)$ & $120.7(5)$ & $C(15)-C(14)-F(1)$ & $119.2(5)$ \\
\hline$N(4)-C(6)-C(5)$ & $122.2(5)$ & $C(15)-C(14)-C(13)$ & $122.2(5)$ \\
\hline$N(3)-C(7)-N(2)$ & $124.0(5)$ & $\mathrm{F}(1)-\mathrm{C}(14)-\mathrm{C}(13)$ & $118.5(5)$ \\
\hline$N(3)-C(7)-C(2)$ & $130.8(5)$ & $\mathrm{C}(14)-\mathrm{C}(15)-\mathrm{N}(5)$ & $121.6(5)$ \\
\hline$N(2)-C(7)-C(2)$ & 105. $2(4)$ & $\mathrm{C}(14)-\mathrm{C}(15)-\mathrm{H}(15 \mathrm{~A})$ & 119.2 \\
\hline$N(4)-C(8)-C(9)$ & $112.2(6)$ & $N(5)-C(15)-H(15 A)$ & 119.2 \\
\hline $\mathrm{N}(4)-\mathrm{C}(8)-\mathrm{H}(8 \mathrm{~A})$ & 109.2 & $\mathrm{~N}(5)-\mathrm{C}(16)-0(3)$ & $118.0(5)$ \\
\hline $\mathrm{C}(9)-\mathrm{C}(8)-\mathrm{H}(8 \mathrm{~A})$ & 109.2 & $N(5)-C(16)-C(12)$ & $124.0(5)$ \\
\hline $\mathrm{N}(4)-\mathrm{C}(8)-\mathrm{H}(8 \mathrm{~B})$ & 109. 2 & $0(3)-C(16)-C(12)$ & $117.9(4)$ \\
\hline $\mathrm{C}(9)-\mathrm{C}(8)-\mathrm{H}(8 \mathrm{~B})$ & 109.2 & $0(3)-C(17)-C(18)$ & 106. $8(5)$ \\
\hline $\mathrm{H}(8 \mathrm{~A})-\mathrm{C}(8)-\mathrm{H}(8 \mathrm{~B})$ & 107.9 & $0(3)-\mathrm{C}(17)-\mathrm{H}(17 \mathrm{~A})$ & 110.4 \\
\hline $0(2)-C(9)-C(8)$ & $112.1(5)$ & $\mathrm{C}(18)-\mathrm{C}(17)-\mathrm{H}(17 \mathrm{~A})$ & 110.4 \\
\hline $0(2)-C(9)-H(9 A)$ & 109.2 & $0(3)-C(17)-H(17 B)$ & 110.4 \\
\hline$C(8)-C(9)-H(9 A)$ & 109. 2 & $\mathrm{C}(18)-\mathrm{C}(17)-\mathrm{H}(17 \mathrm{~B})$ & 110.4 \\
\hline $0(2)-C(9)-H(9 B)$ & 109.2 & $\mathrm{H}(17 \mathrm{~A})-\mathrm{C}(17)-\mathrm{H}(17 \mathrm{~B})$ & 108.6 \\
\hline $\mathrm{C}(8)-\mathrm{C}(9)-\mathrm{H}(9 \mathrm{~B})$ & 109.2 & $\mathrm{~N}(6)-\mathrm{C}(18)-\mathrm{C}(19)$ & $118.8(6)$ \\
\hline $\mathrm{H}(9 \mathrm{~A})-\mathrm{C}(9)-\mathrm{H}(9 \mathrm{~B})$ & 107.9 & $N(6)-C(18)-C(20)$ & $121.8(6)$ \\
\hline $0(2)-C(10)-C(11)$ & $111.9(5)$ & $C(19)-C(18)-C(20)$ & 58. 1(6) \\
\hline $0(2)-C(10)-H(10 A)$ & 109.2 & $N(6)-C(18)-C(17)$ & $112.0(5)$ \\
\hline $\mathrm{C}(11)-\mathrm{C}(10)-\mathrm{H}(10 \mathrm{~A})$ & 109.2 & $C(19)-C(18)-C(17)$ & $119.8(6)$ \\
\hline $0(2)-\mathrm{C}(10)-\mathrm{H}(10 \mathrm{~B})$ & 109.2 & $C(20)-C(18)-C(17)$ & $116.7(5)$ \\
\hline $\mathrm{C}(11)-\mathrm{C}(10)-\mathrm{H}(10 \mathrm{~B})$ & 109.2 & $C(20)-C(19)-C(18)$ & $61.3(6)$ \\
\hline $\mathrm{H}(10 \mathrm{~A})-\mathrm{C}(10)-\mathrm{H}(10 \mathrm{~B})$ & 107.9 & $\mathrm{C}(20)-\mathrm{C}(19)-\mathrm{H}(19 \mathrm{~A})$ & 117.6 \\
\hline$N(4)-C(11)-C(10)$ & $110.8(5)$ & $C(18)-C(19)-H(19 A)$ & 117.6 \\
\hline$N(4)-C(11)-C(12)$ & $113.2(4)$ & $\mathrm{C}(20)-\mathrm{C}(19)-\mathrm{H}(19 \mathrm{~B})$ & 117.6 \\
\hline$C(10)-C(11)-C(12)$ & $110.0(4)$ & $\mathrm{C}(18)-\mathrm{C}(19)-\mathrm{H}(19 \mathrm{~B})$ & 117.6 \\
\hline$N(4)-C(11)-H(11 A)$ & 107.5 & $H(19 A)-C(19)-H(19 B)$ & 114.7 \\
\hline$C(10)-C(11)-H(11 A)$ & 107.5 & $C(19)-C(20)-C(18)$ & $60.5(5)$ \\
\hline $\mathrm{C}(12)-\mathrm{C}(11)-\mathrm{H}(11 \mathrm{~A})$ & 107.5 & $C(19)-C(20)-H(20 A)$ & 117.7 \\
\hline$C(13)-C(12)-C(16)$ & $116.7(4)$ & $C(18)-C(20)-H(20 A)$ & 117.7 \\
\hline$C(13)-C(12)-C(11)$ & $121.7(5)$ & $\mathrm{C}(19)-\mathrm{C}(20)-\mathrm{H}(20 \mathrm{~B})$ & 117.7 \\
\hline$C(16)-C(12)-C(11)$ & $121.6(4)$ & $\mathrm{C}(18)-\mathrm{C}(20)-\mathrm{H}(20 \mathrm{~B})$ & 117.7 \\
\hline$C(14)-C(13)-C(12)$ & $117.9(5)$ & $\mathrm{H}(20 \mathrm{~A})-\mathrm{C}(20)-\mathrm{H}(20 \mathrm{~B})$ & 114.8 \\
\hline
\end{tabular}


Table 4. Torsion angles [deg] for LPM4870108.

\begin{tabular}{|c|c|}
\hline $\mathrm{C}(3)-\mathrm{N}(1)-\mathrm{N}(2)-\mathrm{C}(7)$ & $0.9(7)$ \\
\hline $\mathrm{C}(3)-\mathrm{N}(1)-\mathrm{N}(2)-\mathrm{C}(4)$ & 177. $0(6)$ \\
\hline $\mathrm{C}(18)-\mathrm{N}(6)-\mathrm{C}(1)-0(1)$ & 15. $6(10)$ \\
\hline $\mathrm{C}(18)-\mathrm{N}(6)-\mathrm{C}(1)-\mathrm{C}(2)$ & $-163.6(5)$ \\
\hline $0(1)-C(1)-C(2)-C(3)$ & 12. 1(10) \\
\hline$N(6)-C(1)-C(2)-C(3)$ & $-168.7(6)$ \\
\hline $0(1)-C(1)-C(2)-C(7)$ & $-160.9(6)$ \\
\hline$N(6)-C(1)-C(2)-C(7)$ & 18. $3(8)$ \\
\hline$N(2)-N(1)-C(3)-C(2)$ & $-0.7(8)$ \\
\hline $\mathrm{C}(7)-\mathrm{C}(2)-\mathrm{C}(3)-\mathrm{N}(1)$ & $0.3(8)$ \\
\hline $\mathrm{C}(1)-\mathrm{C}(2)-\mathrm{C}(3)-\mathrm{N}(1)$ & $-174.0(6)$ \\
\hline $\mathrm{N}(1)-\mathrm{N}(2)-\mathrm{C}(4)-\mathrm{C}(5)$ & $-179.3(5)$ \\
\hline $\mathrm{C}(7)-\mathrm{N}(2)-\mathrm{C}(4)-\mathrm{C}(5)$ & $-3.3(8)$ \\
\hline$N(2)-C(4)-C(5)-C(6)$ & 1. $0(8)$ \\
\hline $\mathrm{C}(7)-\mathrm{N}(3)-\mathrm{C}(6)-\mathrm{N}(4)$ & $179.6(4)$ \\
\hline $\mathrm{C}(7)-\mathrm{N}(3)-\mathrm{C}(6)-\mathrm{C}(5)$ & $-0.2(6)$ \\
\hline $\mathrm{C}(8)-\mathrm{N}(4)-\mathrm{C}(6)-\mathrm{N}(3)$ & 176. $7(5)$ \\
\hline $\mathrm{C}(11)-\mathrm{N}(4)-\mathrm{C}(6)-\mathrm{N}(3)$ & 10.4(6) \\
\hline $\mathrm{C}(8)-\mathrm{N}(4)-\mathrm{C}(6)-\mathrm{C}(5)$ & $-3.5(7)$ \\
\hline $\mathrm{C}(11)-\mathrm{N}(4)-\mathrm{C}(6)-\mathrm{C}(5)$ & $-169.8(4)$ \\
\hline $\mathrm{C}(4)-\mathrm{C}(5)-\mathrm{C}(6)-\mathrm{N}(3)$ & $0.8(7)$ \\
\hline $\mathrm{C}(4)-\mathrm{C}(5)-\mathrm{C}(6)-\mathrm{N}(4)$ & $-179.0(5)$ \\
\hline $\mathrm{C}(6)-\mathrm{N}(3)-\mathrm{C}(7)-\mathrm{N}(2)$ & $-2.3(7)$ \\
\hline $\mathrm{C}(6)-\mathrm{N}(3)-\mathrm{C}(7)-\mathrm{C}(2)$ & 179. 4(5) \\
\hline $\mathrm{N}(1)-\mathrm{N}(2)-\mathrm{C}(7)-\mathrm{N}(3)$ & $-179.4(5)$ \\
\hline $\mathrm{C}(4)-\mathrm{N}(2)-\mathrm{C}(7)-\mathrm{N}(3)$ & 4. 1(7) \\
\hline$N(1)-N(2)-C(7)-C(2)$ & $-0.8(6)$ \\
\hline$C(4)-N(2)-C(7)-C(2)$ & $-177.3(5)$ \\
\hline $\mathrm{C}(3)-\mathrm{C}(2)-\mathrm{C}(7)-\mathrm{N}(3)$ & 178. 8(5) \\
\hline$C(1)-C(2)-C(7)-N(3)$ & $-6.9(9)$ \\
\hline $\mathrm{C}(3)-\mathrm{C}(2)-\mathrm{C}(7)-\mathrm{N}(2)$ & $0.3(6)$ \\
\hline $\mathrm{C}(1)-\mathrm{C}(2)-\mathrm{C}(7)-\mathrm{N}(2)$ & $174.6(5)$ \\
\hline $\mathrm{C}(6)-\mathrm{N}(4)-\mathrm{C}(8)-\mathrm{C}(9)$ & $161.9(5)$ \\
\hline $\mathrm{C}(11)-\mathrm{N}(4)-\mathrm{C}(8)-\mathrm{C}(9)$ & $-32.0(7)$ \\
\hline $\mathrm{C}(10)-0(2)-\mathrm{C}(9)-\mathrm{C}(8)$ & $-65.8(7)$ \\
\hline $\mathrm{N}(4)-\mathrm{C}(8)-\mathrm{C}(9)-0(2)$ & 48. $1(8)$ \\
\hline $\mathrm{C}(9)-0(2)-\mathrm{C}(10)-\mathrm{C}(11)$ & $66.2(7)$ \\
\hline $\mathrm{C}(6)-\mathrm{N}(4)-\mathrm{C}(11)-\mathrm{C}(10)$ & $-161.4(4)$ \\
\hline $\mathrm{C}(8)-\mathrm{N}(4)-\mathrm{C}(11)-\mathrm{C}(10)$ & $32.0(6)$ \\
\hline $\mathrm{C}(6)-\mathrm{N}(4)-\mathrm{C}(11)-\mathrm{C}(12)$ & 74. 4(5) \\
\hline $\mathrm{C}(8)-\mathrm{N}(4)-\mathrm{C}(11)-\mathrm{C}(12)$ & $-92.1(6)$ \\
\hline $0(2)-\mathrm{C}(10)-\mathrm{C}(11)-\mathrm{N}(4)$ & $-48.3(6)$ \\
\hline
\end{tabular}




$$
\begin{array}{lr}
0(2)-\mathrm{C}(10)-\mathrm{C}(11)-\mathrm{C}(12) & 77.7(6) \\
\mathrm{N}(4)-\mathrm{C}(11)-\mathrm{C}(12)-\mathrm{C}(13) & 51.6(6) \\
\mathrm{C}(10)-\mathrm{C}(11)-\mathrm{C}(12)-\mathrm{C}(13) & -72.9(6) \\
\mathrm{N}(4)-\mathrm{C}(11)-\mathrm{C}(12)-\mathrm{C}(16) & -131.0(5) \\
\mathrm{C}(10)-\mathrm{C}(11)-\mathrm{C}(12)-\mathrm{C}(16) & 104.5(5) \\
\mathrm{C}(16)-\mathrm{C}(12)-\mathrm{C}(13)-\mathrm{C}(14) & -0.1(7) \\
\mathrm{C}(11)-\mathrm{C}(12)-\mathrm{C}(13)-\mathrm{C}(14) & 177.4(5) \\
\mathrm{C}(12)-\mathrm{C}(13)-\mathrm{C}(14)-\mathrm{C}(15) & -1.6(8) \\
\mathrm{C}(12)-\mathrm{C}(13)-\mathrm{C}(14)-\mathrm{F}(1) & 177.8(5) \\
\mathrm{F}(1)-\mathrm{C}(14)-\mathrm{C}(15)-\mathrm{N}(5) & -178.5(5) \\
\mathrm{C}(13)-\mathrm{C}(14)-\mathrm{C}(15)-\mathrm{N}(5) & 0.9(9) \\
\mathrm{C}(16)-\mathrm{N}(5)-\mathrm{C}(15)-\mathrm{C}(14) & 1.7(8) \\
\mathrm{C}(15)-\mathrm{N}(5)-\mathrm{C}(16)-0(3) & 178.4(5) \\
\mathrm{C}(15)-\mathrm{N}(5)-\mathrm{C}(16)-\mathrm{C}(12) & -3.6(7) \\
\mathrm{C}(17)-0(3)-\mathrm{C}(16)-\mathrm{N}(5) & -25.7(7) \\
\mathrm{C}(17)-0(3)-\mathrm{C}(16)-\mathrm{C}(12) & 156.2(5) \\
\mathrm{C}(13)-\mathrm{C}(12)-\mathrm{C}(16)-\mathrm{N}(5) & 2.8(7) \\
\mathrm{C}(11)-\mathrm{C}(12)-\mathrm{C}(16)-\mathrm{N}(5) & -174.7(4) \\
\mathrm{C}(13)-\mathrm{C}(12)-\mathrm{C}(16)-0(3) & -179.2(4) \\
\mathrm{C}(11)-\mathrm{C}(12)-\mathrm{C}(16)-0(3) & 3.3(6) \\
\mathrm{C}(16)-0(3)-\mathrm{C}(17)-\mathrm{C}(18) & -145.4(5) \\
\mathrm{C}(1)-\mathrm{N}(6)-\mathrm{C}(18)-\mathrm{C}(19) & -108.3(9) \\
\mathrm{C}(1)-\mathrm{N}(6)-\mathrm{C}(18)-\mathrm{C}(20) & -39.9(10) \\
\mathrm{C}(1)-\mathrm{N}(6)-\mathrm{C}(18)-\mathrm{C}(17) & 105.2(7) \\
0(3)-\mathrm{C}(17)-\mathrm{C}(18)-\mathrm{N}(6) & 55.2(7) \\
0(3)-\mathrm{C}(17)-\mathrm{C}(18)-\mathrm{C}(19) & -91.0(7) \\
0(3)-\mathrm{C}(17)-\mathrm{C}(18)-\mathrm{C}(20) & -157.8(7) \\
\mathrm{N}(6)-\mathrm{C}(18)-\mathrm{C}(19)-\mathrm{C}(20) & 111.4(7) \\
\mathrm{C}(17)-\mathrm{C}(18)-\mathrm{C}(19)-\mathrm{C}(20) & -104.6(8) \\
\mathrm{N}(6)-\mathrm{C}(18)-\mathrm{C}(20)-\mathrm{C}(19) & -106.4(8) \\
\mathrm{C}(17)-\mathrm{C}(18)-\mathrm{C}(20)-\mathrm{C}(19) & 110.1(8) \\
& \\
&
\end{array}
$$

Table 5. Hydrogen bonds for LPM4870108 [A and deg.].

\begin{tabular}{lllll}
\hline $\mathrm{D}-\mathrm{H} \ldots \mathrm{A}$ & $\mathrm{d}(\mathrm{D}-\mathrm{H})$ & $\mathrm{d}(\mathrm{H} . \ldots \mathrm{A})$ & $\mathrm{d}(\mathrm{D} \ldots \mathrm{A})$ & $<(\mathrm{DHA})$ \\
$\mathrm{N}(6)-\mathrm{H}(6 \mathrm{~A}) \ldots \mathrm{N}(3)$ & 0.86 & 2.29 & $2.899(6)$ & 128.0 \\
& & & & \\
\hline
\end{tabular}

